\title{
High Throughput Screening at the Membrane Interface Reveals New Inhibitors of Amyloid- $\beta$
}

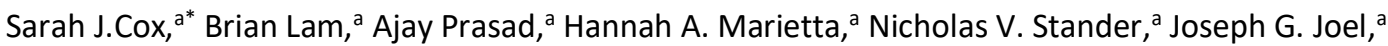

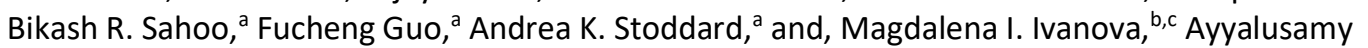 \\ Ramamoorthy ${ }^{\mathrm{a}, \mathrm{c}^{*}}$
}

\begin{abstract}
Amyloid- $\beta$ aggregation at the cell-membrane of neruonal cells is implicated as a source of toxicity for Alzheimer's disease. Small molecules have been studied for their ability to supress amyloid aggregation and toxicity, but the presence of membranes negate their activity. Here, we have identified $\mathbf{5}$ small molecules that are active at the membrane interface.
\end{abstract}

Alzheimer's disease (AD) is a deadly and debilitating ailment that currently affects 50 million people worldwide. ${ }^{1}$ Early research into $A D$ focused on the presence of indicative protein amyloid-beta $(A \beta)$ fibrils due to their prominence in postmortem examination of patients' brains. However, it is now hypothesized that small, toxic, intermediate species, known as oligomers, are the predominant toxic amyloid-beta $\left(A \beta_{40}\right)$ species in $A D .^{2} A \beta$ peptides are produced from the cleavage of amyloid precursor protein (APP) in the extracellular membrane by $\beta$ and $\gamma$-secretases. Cellular membranes have been implicated to be a site of potential toxicity and can act as a catalyst for amyloid aggregation. ${ }^{3}$ Some oligomers are proposed to impart their toxic function by interacting directly with the cell membrane of neurons then disrupting and permeabilizing the membrane. As a result, non-selective ion channels and large pores are created which, in turn, ablate the charge gradient necessary for neuronal function. ${ }^{4}$ Many studies suggest that lipid membranes are able to accelerate the aggregation of $A \beta$ as well as facilitate the formation of unique structures of $A \beta$ species that are specific to lipid bilayer disruption. ${ }^{5}$

There has been extensive investigation into small molecules with the ability to modulate the aggregation of $A \beta$ in solution. ${ }^{6}$ However, the search for modulators of $A \beta$ aggregation has relied heavily on serendipity; often times, a novel class of

\footnotetext{
a. Department of Chemistry, University of Michigan, Ann Arbor, MI (USA) email: coxsj@umich.edu,ramamoor@umich.edu

b. Department of Neurology, University of Michigan, Ann Arbor, MI, 48109, USA

c. Biophysics Program, University of Michigan, Ann Arbor, MI 48109 U.S.A

Electronic Supplementary Information (ESI) available: See DOI: 10.1039/x0xx00000x
}

inhibitors is accidentally discovered, and improved analogues are subsequently synthesized. ${ }^{7}$ Relying on accidental discoveries is unlikely to generate a diverse enough chemical portfolio to successfully generate a drug candidate that can demonstrate clinical efficacy. Thus, it is essential to identify new and novel chemical species which may be specifically capable of modulating membrane-assisted $A \beta_{40}$ aggregation for use as toxic $A \beta$ oligomer probes. ${ }^{2}$ Here, through the usage of a small molecule library, 5 compounds have been identified that modulate the formation of $A \beta_{40}$ aggregates in the presence of lipid membrane. These small molecules represent an avenue for the development and further investigation of $A \beta_{40}$ and membrane interactions.

Using a library of over 1,800 compounds, selected for their chemical diversity and biological activity, the screening was performed by the addition of biologically obtained $A \beta_{40}$ in the presence of large unilamellar vesicles (LUVs) composed of a mixture of 7:3 molar ratio of DOPC:DOPG, which represents the charge distribution of eukaryotic membranes (Figure 1A). To probe the interactions between the lipid bilayer, small molecules, and $A \beta_{40}$, we used a fluorescence readout assay regularly employed in amyloid studies using Thioflavin-T (ThT) dye. ThT assays provide insights into the kinetics of amyloid formation, which facilitates the identification of compounds that are able to inhibit the formation of $\beta$-sheet rich amyloid aggregates. Signal intensity can be proportional to the fibers present and decreases in intensity can be indicative of a decrease in overall fiber content (Figure S1). We optimized screening conditions, achieving a Z-score of 0.46 (Figure 1C). 40 reproducible hits were selected after ruling out initially fluorescent compounds and compounds which did not give a matching read-out in twin sets of plates. These 40 compounds were then used for a concentration response curve (CRC) titration screen to determine the activity of the compounds in a range of concentrations (Figures S2 and S3). Results from the CRC screen helped us to narrow down the 40 initial hits to 21 primary hits based on the calculated IC50 values and the 

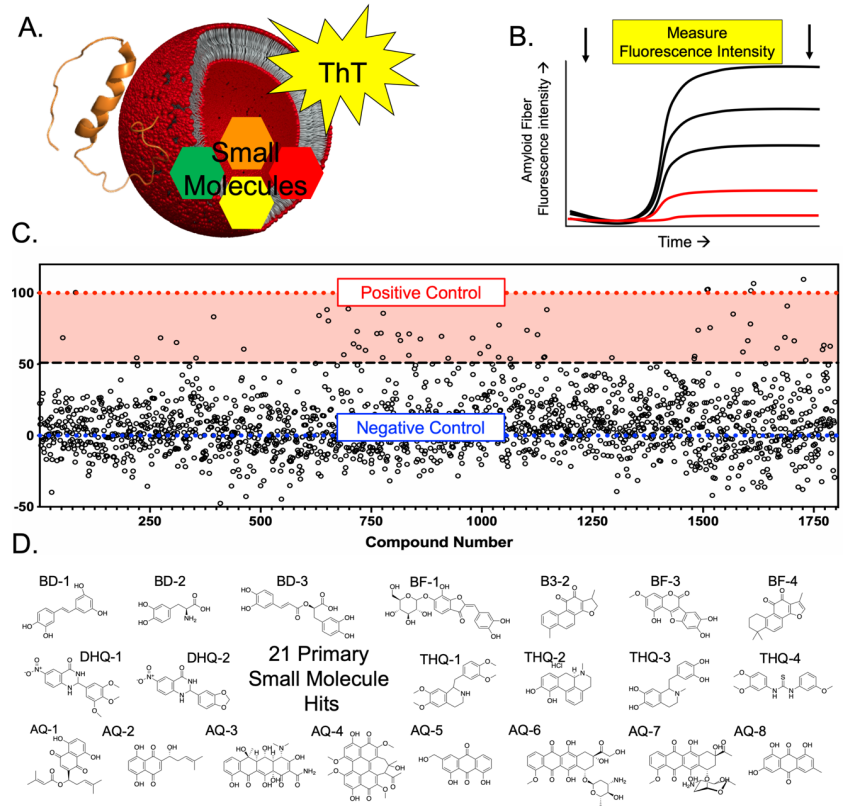

Figure 1. (A) An illustration of the components of the screen: $A \beta_{40}$ monomer (2LFM), LUVs, small molecules and ThT. (B) schematic of reading the assay plates before aggregation and then heating and shaking the plates before reading the final fluorescence intensity after 24 hours of incubation. (C) Final fluorescent intensity of every compounded screened for inhibition. Value of twin plates are averaged and normalized in respect to the positive and negative controls. (D) 21 primary small molecule hits chosen after initial screen and CRC testing.

exclusion of known compounds with PAINS properties (Figure 1D, Tables S1 and S2). ${ }^{7}$

The selected 21 compounds were initially subjected to full ThT kinetic profiles with measurements taken every 5 minutes (Figure S4). Each compound was tested at 10, 5 and 2 molar equivalencies in respect to the concentration of $A \beta_{40}$ while in the presence of $500 \mu \mathrm{M}$ LUVs. Initially by the ThT assay, many appear to be promising and robust inhibitors, with many compounds negating the aggregation fully at all the concentrations tested. However, while as critical as the ThT assay is to studying amyloid aggregation, it is also subject to fluorescent quenching, overlap, or displacement by other compounds. Because of this, secondary confirmation not relying on fluorescence was performed to further narrow down the hit compounds. To do this we used the dot blot assay utilizing the $O C$ anti-amyloid fiber antibody, which is known to bind to the general amyloid fiber $\beta$-sheet epitope (Figures 2A and S5). ${ }^{9}$ While many of the compounds looked to be complete inhibitors by the ThT assay, the strong antibody reactivity observed for $A \beta$ in presence of these compounds indicated that they do not inhibit fiber formation. After identifying compounds that interfere with ThT, we then examined the compounds that gave $50 \%$ or less reactivity by the dot blot assay by examining them via Transmission Electron Microscopy (TEM) (Figure 2B). Out of the 15 compounds investigated, 5 of them inhibited $A \beta_{40}$ fibers, thus AQ-4, THQ-1, BF-3, DHQ-1 and DHQ-2, which were selected for a deeper investigation. Of the other 10 compounds that had fiber formation identified at this stage, many of them exhibited very interesting and distinct fiber morphologies, which could be of interest for further investigation, as some of them have been reported to be amyloid inhibitors in the absence of membrane.

Understanding the secondary structure transitions is important for amyloid investigation, as there is a known shift from a random-coil monomer to $\beta$-sheet fiber. To study the compounds' effects on $A \beta$ secondary structure, the 5 nonwater-soluble compounds were incorporated in the lipid bilayer, which was confirmed by UV-Vis (Figure S6). Upon incorporation of the compounds in the lipid bilayer at a 10:1 lipid to compound molar ratio, we monitored the $A \beta$ structural transitions by circular dichroism (CD) experiments as well as by ThT kinetics (Figures 2C, S7, S8). After 24 hours, AQ-4 and BF-3 still exhibited a random-coil structure. DHQ-2 and THQ-1 showed a minor helical conformation, while DHQ-1 showed a strong $\beta$-sheet conformation for $A \beta$. Up to 7 days, $A Q-4$ maintained random-coil conformation, whereas BF-3, DHQ-1, and DHQ-2 showed $\beta$-sheet conformation. THQ-1, however, showed poor signal and showed some slight $\beta$-sheet characteristics for $A \beta$ but was not fully interpretable at 48 hours, but appeared more clearly $\beta$-sheet after 7 days.

Using NMR, we investigated the interaction of ${ }^{15} \mathrm{~N}$-labeled$A \beta_{40}$ with the reported compounds both with and without the presence of loaded LUVs using $2 \mathrm{D}{ }^{1} \mathrm{H} /{ }^{15} \mathrm{~N}$ SOFAST-HMQC experiments (Figures 3, S9, and S10). This experiment is useful in determining the level of peptide aggregation (or monomer depletion) in solution. Because large aggregates such as amyloid fibers tumble slowly on the NMR timescale they do not contribute to the observed signal and any signal is conferred to be fast tumbling oligomers or monomers. The volume of each peak as well as the signal-to-noise ratio were analyzed (Figures 3 and S11-S13). In the presence of loaded vesicles, $A \beta_{40}$ showed 15 well resolved peaks at time zero, with only 4 peaks with poor $\mathrm{S} / \mathrm{N}$ were observable after 96 hours. For three of the compounds (DHQ-1, DHQ-2 and BF-3), the observed NMR resonances was found to be distributed throughout the amino acid sequence of $A \beta_{40}$ at time zero and after 24 hours, which

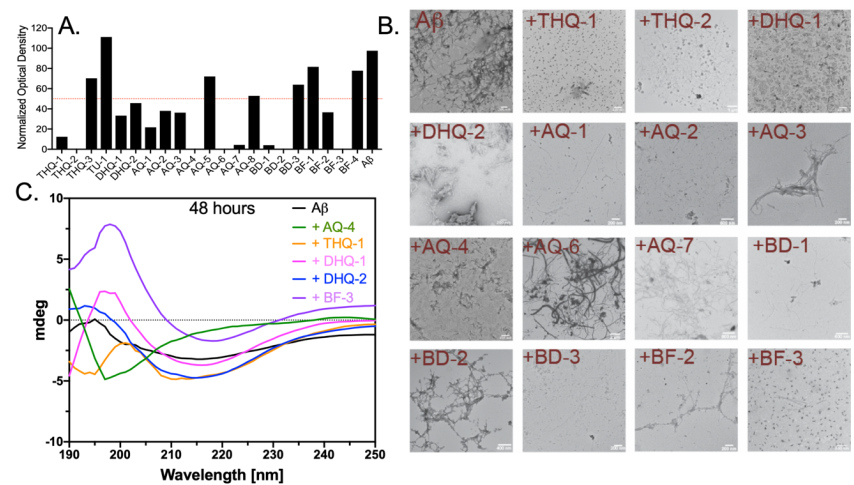

Figure 2. (A) Signal intensities from dot blot assay using the $O C$ antibody. Samples were measured after ThT experiments with 5 equivalents of a compound with respect to $A \beta_{40}$. (B) TEM images for compounds that gave less than $50 \%$ antibody reactivity. (C) $C D$ spectra of $25 \mu \mathrm{M} \mathrm{A} \beta_{40}$ in the presence of $500 \mu \mathrm{M}$ of LUVs with 50 $\mu \mathrm{M}$ loaded compound after 48 hours of incubation with background subtraction of loaded LUVs. 
was also the case in the samples without lipids. For AQ-4, 5 peaks from the $C$ terminus of $A \beta_{40}$ were seen even after 96 hours of incubation, which was also seen in the sample without lipids, indicating some sort of conserved similarities which could signify that the compound is both interacting with the lipid bilayer as well as $A \beta_{40}$ itself. A possible explanation to this is that the $\mathrm{N}$ terminus is bound inside an oligomer or to the membrane with a solvent exposed $C$ terminus tail. Spectra of $A \beta$ with THQ1 and lipids showed very little signal intensity at time zero, with no visible peaks after 96 hours. The opposite was seen in the THQ-1 sample without lipids, in which well resolved peaks of $A \beta_{40}$ were seen at both time zero and at 96 hours. This indicates that ThQ1 interacts and inhibits $A \beta_{40}$ aggregation but does not interact well in presence of lipids, at least when loaded with lipids, since it appeared to be a strong inhibitor in the assays prior to loading the compounds in LUVs. It is also probable that some of the signal loss and poor $S / N$ could be due to $A \beta_{40}$ binding to $100 \mathrm{~nm}$ LUVs that decreases the tumbling rate of LUVs. Experiments utilizing smaller membrane mimetic such as nanodiscs and implementing paramagnetic quenching NMR experiments could be an enlightening next step to understand these systems.

After the NMR measurements, the samples in presence of lipids were analyzed using Size Exclusion Chromatography (SEC) (Figures 4 and S14). Three distinct peak areas were seen and the area under each curve was quantified to determine the distribution of aggregates within the sample. Peak area 1
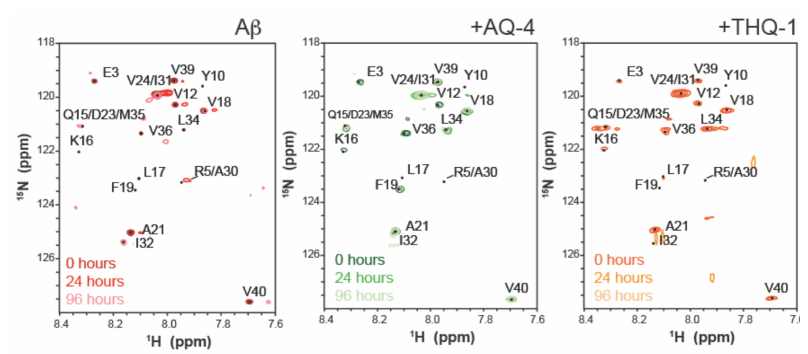

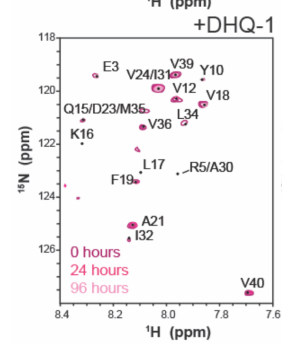

$\mathbf{A} \beta$
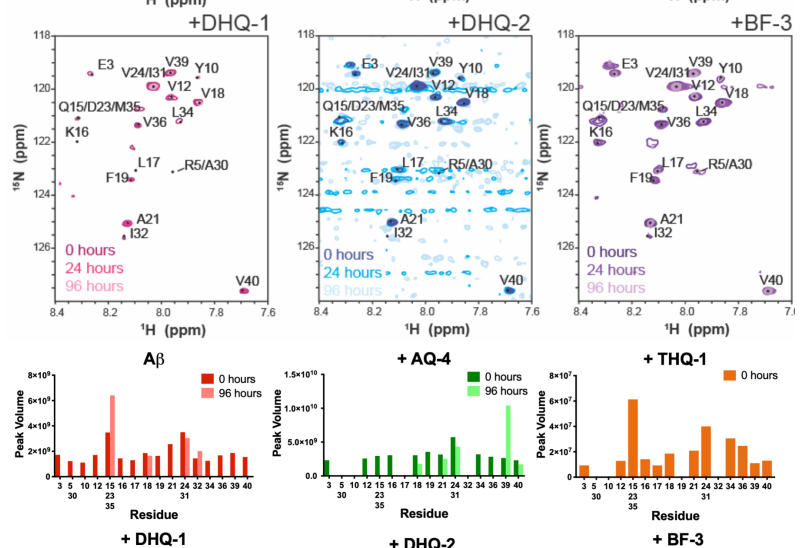

+ THQ-1
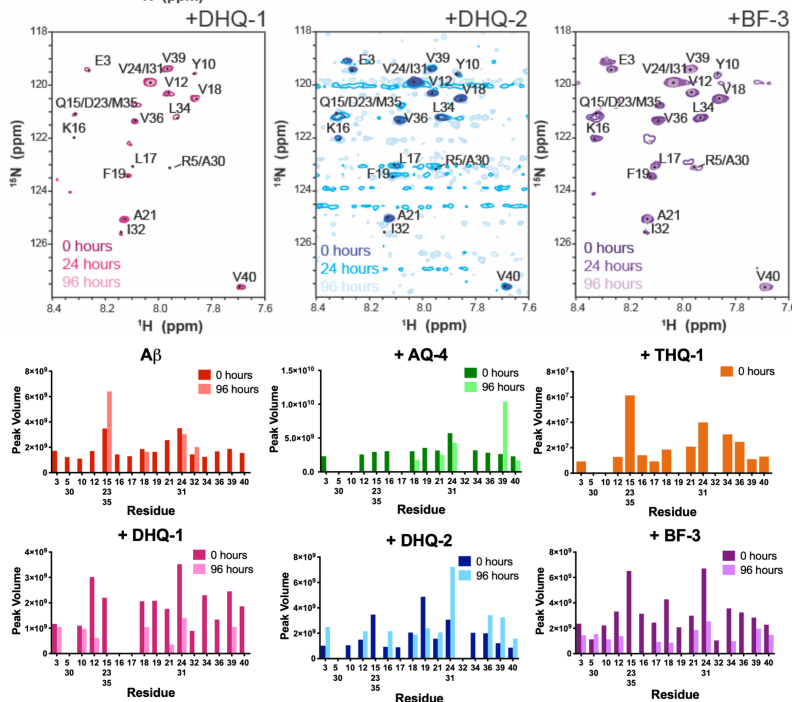

Figure 3. (top 2 rows) SOFAST-HMQC NMR spectra of $25 \mu \mathrm{M}$ ${ }^{15} \mathrm{~N}$-labeled-A $\beta_{40}$ in the presence of $500 \mu \mathrm{M}$ of LUVs with a 50 $\mu \mathrm{M}$ loaded compound at 0,24 and 96 hours. (bottom 2 rows) Peak volume of visible peaks at 0 and 96 hours.

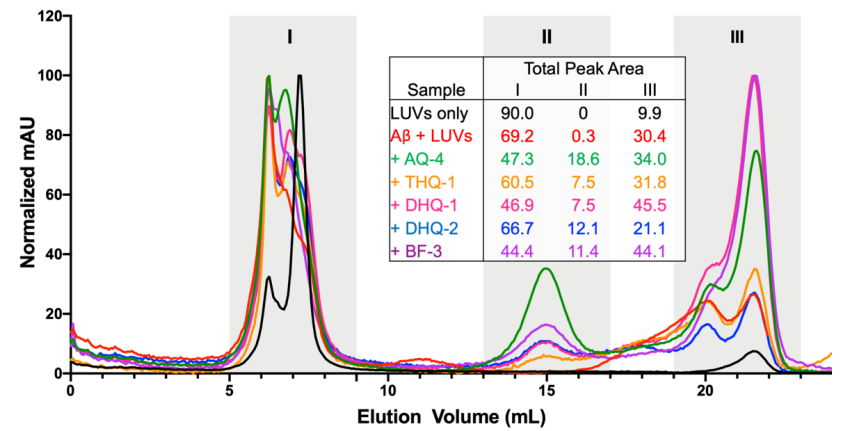

Figure 4. Size exclusion chromatography of the indicated NMR samples and normalized area under the curve of each peak area measured for all samples.

(between 5 and $8 \mathrm{~mL}$ ) consisted of multiple peaks most likely made up of LUVs and $A \beta_{40}$ amyloid fibers. Peak area $2(15 \mathrm{~mL})$ most possibly corresponds to an oligomer of 4 monomers ( 17 $\mathrm{kDa}$ ) or an oligomer of 3 monomers with each bound to 2 compounds ( $16 \mathrm{kDa}$ ). Lastly, Peak area 3 could correspond to monomer and dimer of $A \beta_{40}$, eluting at 21.5 and $20 \mathrm{~mL}$ respectively. Since, these samples contain lipids, it is possible that a portion of the intensity of the peaks is also lipids that have been fragmented from the LUVs. For AQ-4, DHQ-1 and BF-3, over $50 \%$ of the eluted sample was either in peak area 2 or 3 , indicating the presence of a small amount of fibers. While the control, THQ-1, and DHQ-2 samples had $60 \%$ or more of the total signal in peak area 1 . The control samples showed no signal inside of peak area 2. Additionally, there was a very small peak seen at $11 \mathrm{~mL}$ which could indicate a very large oligomer or protofibers. Inside peak area 1 , three distinct peaks were observed: one for LUVs at $7.5 \mathrm{~mL}$, one for fibers at $6.5 \mathrm{~mL}$, and in between them; there is also a less resolved peak that may be $A \beta_{40}$ bound to LUVs, which is also not well resolved with the control sample of $A \beta_{40}$ in LUVs. For the control LUVs sample, a small percentage of the total sample eluted in peak area 3 , which could correspond to small lipid micelles. This could indicate that some of the signal in the other samples may also contain micelles or lipids that have been fragmented from the bilayer as the result of the peptide aggregation on the membrane.

\section{Conclusions}

A high-throughput screen has led to the identification of 5 membrane active $A \beta_{40}$ amyloid inhibitors, with a brief summary in Table 1. Among them, DHQ-1, DHQ-2 and THQ-1 were found to be the least robust as shown by NMR, SEC and CD results, whereas BF-3 and $A Q-4$ exhibited the most evidence that they are able to stop the aggregation (or trap the aggregates) at the membrane interface. AQ4 showed the highest ratio of oligomers by SEC, a constant randomcoil signal from $C D$ as well as the same 5 residues maintaining signal intensity in samples with and without membrane. This could indicate that even though AQ-4 interacts with the membrane, it is also able to directly interact with $A \beta_{40}$. BF3 showed similar results as that observed for AQ-4. It showed no aggregation by ThT, maintained observable NMR signal intensity but induced an overall loss in intensity for every residue and also showed smaller species by SEC; 


\begin{tabular}{|c|c|c|c|c|c|c|}
\hline \multirow[b]{2}{*}{ Compound } & \multicolumn{6}{|c|}{ Biophysical Technique } \\
\hline & ThT & CD & NMR & Dot Blot & TEM & SEC \\
\hline & $\begin{array}{l}\text { Complete } \\
\text { inhibition }\end{array}$ & $\begin{array}{c}\text { Random } \\
\text { coil }\end{array}$ & $\begin{array}{c}\text { C-terminus } \\
\text { visible }\end{array}$ & No binding & $\begin{array}{l}\text { Amorphous, } \\
\text { no fibers }\end{array}$ & $\begin{array}{l}>50 \% \text { in } \\
\text { peaks IIIIII }\end{array}$ \\
\hline & $\begin{array}{l}\text { Abnormal } \\
\text { curve }\end{array}$ & $\begin{array}{c}\text { Weak } \\
\text { beta } \\
\text { sheet } \\
\text { /mixture }\end{array}$ & No visible peaks & $15 \%$ binding & $\begin{array}{c}\text { Small } \\
\text { particles, few } \\
\text { fibers }\end{array}$ & $\begin{array}{l}>60 \% \text { in } \\
\text { peak I }\end{array}$ \\
\hline & $\begin{array}{l}\text { Complete } \\
\text { inhibition }\end{array}$ & $\begin{array}{l}\text { Beta } \\
\text { sheet }\end{array}$ & $\begin{array}{l}\text { Peaks missing } \\
\text { in core, SN } \\
\text { ratio maintained }\end{array}$ & $30 \%$ binding & $\begin{array}{c}\text { Small } \\
\text { particles, few } \\
\text { fibers }\end{array}$ & $\begin{array}{l}>50 \% \text { in } \\
\text { peaks II/III }\end{array}$ \\
\hline & $\begin{array}{c}\text { Partial } \\
\text { inhibition }\end{array}$ & $\begin{array}{l}\text { Beta } \\
\text { sheet }\end{array}$ & $\begin{array}{l}\text { Peaks remain } \\
\text { visible, S/N ratio } \\
\text { maintained }\end{array}$ & $40 \%$ binding & $\begin{array}{l}\text { Amorphous, } \\
\text { few fibers }\end{array}$ & $\begin{array}{l}>60 \% \text { in } \\
\text { peak I }\end{array}$ \\
\hline & $\begin{array}{l}\text { Complete } \\
\text { inhibition }\end{array}$ & $\begin{array}{l}\text { Beta } \\
\text { sheet }\end{array}$ & $\begin{array}{l}\text { Peaks remain } \\
\text { visible, S/N ratio } \\
\text { decreased }\end{array}$ & No binding & $\begin{array}{c}\text { Small } \\
\text { particles, few } \\
\text { fibers }\end{array}$ & $\begin{array}{c}>50 \% \text { in } \\
\text { peaks II/III }\end{array}$ \\
\hline
\end{tabular}

Table 1. Summary of findings from the biophysical characterization of amyloid inhibition by the 5 compounds.

this observation could indicate that while BF3 does not allow $A \beta_{40}$ to aggregate, some $A \beta_{40}$ population is still binding to the membrane surface. While DHQ-1 DHQ-2 and THQ-1 are less membrane active, they are still novel scaffolds for the inhibition of $A \beta_{40}$. Although DHQ1 and DHQ-2 showed some differences in activity, they may be a good starting point for developing derivatives. Given their similar architecture and sites for potential for synthesis, they could be used in an interesting study for structure activity relationship (SAR) analysis. The initial investigation and subsequent rule out of THQ-1 demonstrates the need for deep characterization for amyloid and small molecule interactions.

Given the similarity of membrane activities of AQ- 4 and BF-3, it is possible that this may be due to their planar structure and lack of free rotation among the aromatic groups. However, many compounds initially investigated as part of the 21 primary hits produced prominent fibers; so clearly there must be unique properties possessed by these two compounds and their interplay between $A \beta_{40}$ and the membrane which render their inhibitory activities. With the presented results as a starting point, NMR would be a robust tool to further investigate the structure of the $A \beta_{40}$ compound structure as well as in conjunction with the membrane.

\section{Conflicts of interest}

There are no conflicts to declare.

\section{Acknowledgments}

This study was supported by the NIH (AG048934 to A.R.)) and the Michigan Alzheimer's disease center MADC (M.I.I). We thank Steve Vander Roest and the University of Michigan Center for Chemical Genomics HTS core.

\section{References}

1 I. W. Hamley, Chem. Rev., 2012, 112, 5147-92.

2 I. Benilova, E. Karran and B. De Strooper, Nat. Neurosci., 2012, 15, 349-357.

3 S. A. Kotler, P. Walsh, J. R. Brender and A. Ramamoorthy,
Chem. Soc. Rev., 2014, 43, 6692-6700. M. S. Terakawa, H. Yagi, M. Adachi, Y.-H. Lee and Y. Goto, J. Biol. Chem., 2015, 290, 815-826.

M. F. M. Sciacca, S. A. Kotler, J. R. Brender, J. Chen, D.-K. Lee and A. Ramamoorthy, Biophys. J., 2012, 103, 702-710.W. Qiang, R. D. Akinlolu, M. Nam and N. Shu, Biochemistry, 2014, 53, 7503-7514.R. D. Johnson, D. G. Steel and A. Gafni, Protein Sci., 2014, 23, 869-83.A. Quist, I. Doudevski, H. Lin, R. Azimova, D. Ng, B. Frangione, B. Kagan, J. Ghiso and R. Lal, Proc. Natl. Acad. Sci. U. S. A., 2005, 102, 10427-32.S. M. Butterfield and H. A. Lashuel, Angew. Chemie - Int. Ed., 2010, 49, 5628-5654. K. J. Korshavn, A. Bhunia, M. H. Lim and A. Ramamoorthy, Chem. Commun., 2016, 52, 882-885.

K. J. Korshavn, C. Satriano, Y. Lin, R. Zhang, M. Dulchavsky, A. Bhunia, M. I. Ivanova, Y.-H. Lee, C. La Rosa, M. H. Lim and A. Ramamoorthy, J. Biol. Chem., 2017, 292, 4638-4650.S. A. Kotler, P. Walsh, J. R. Brender and A. Ramamoorthy, Chem. Soc. Rev., 2014, 43, 6692-6700.

A. R. A. Ladiwala, J. S. Dordick and P. M. Tessier, J. Biol. Chem., 2011, 286, 3209-3218.J. Bieschke, M. Herbst, T. Wiglenda, R. P. Friedrich, A. Boeddrich, F. Schiele, D. Kleckers, J. M. Lopez del Amo, B. a Grüning, Q. Wang, M. R. Schmidt, R. Lurz, R. Anwyl, S. Schnoegl, M. Fändrich, R. F. Frank, B. Reif, S. Günther, D. M. Walsh and E. E. Wanker, Nat. Chem. Biol., 2011, 8, 93-101. J. L. Cummings, T. Morstorf and K. Zhong, .R. Ahmed and G. Melacini, Chem. Commun., 2018, 54, 4644-4652.M. Necula, R. Kayed, S. Milton and C. G. Glabe, J. Biol. Chem., 2007, 282, 1031110324.H. Kroth, A. Ansaloni, Y. Varisco, A. Jan, N. Sreenivasachary, N. Rezaei-Ghaleh, V. Giriens, S. Lohmann, M. P. López-Deber, O. Adolfsson, M. Pihlgren, P. Paganetti, W. Froestl, L. Nagel-Steger, D. Willbold, T. Schrader, M. Zweckstetter, A. Pfeifer, H. A. Lashuel and A. Muhs, J. Biol. Chem., 2012, 287, 34786-34800.J.-S. Choi, J. J. Braymer, R. P. R. Nanga, A. Ramamoorthy and M. H. Lim, .V. Patel, X. Zhang, N. A. Tautiva, A. N. Nyabera, O. O. Owa, M. Baidya, H. C. Sung, P. S. Taunk, S. Abdollahi, S. Charles, R. A. Gonnella, N. Gadi, K. T. Duong, J. N. Fawver, C. Ran, T. O. Jalonen and I. V. J. Murray, Curr. Alzheimer Res., 2015, 12, 445-461.S. Lee, X. Zheng, J. Krishnamoorthy, M. G. Savelieff, H. M. Park, J. R. Brender, J. H. Kim, J. S. Derrick, A. Kochi, H. J. Lee, C. Kim, A. Ramamoorthy, M. T. Bowers and M. H. Lim, J. Am. Chem. Soc., 2014, 136, 299-310.A. J. Doig and P. Derreumaux, Curr. Opin. Struct. Biol., 2015, 30, 50-56.L. M. Young, J. C. Saunders, R. A. Mahood, C. H. Revill, R. J. Foster, L.-H. Tu, D. P. Raleigh, S. E. Radford and A. E. Ashcroft, Nat. Chem., 2015, 7, 73-81.L. M. Young, A. E. Ashcroft and S. E. Radford, Curr. Opin. Chem. Biol., 2017, 39, 90-99.

S. Brahmachari, A. Paul, D. Segal and E. Gazit, Future Med. Chem., 2017, 9, 797-810.

J. Baell and M. A. Walters, Nature, 2014, 513, 481-483.

R. Kayed, E. Head, F. Sarsoza, T. Saing, C. W. Cotman, M. Necula, L. Margol, J. Wu, L. Breydo, J. L. Thompson, S. Rasool, T. Gurlo, P. Butler and C. G. Glabe, Mol. Neurodegener., 2007, 2, 18. 